\title{
Research on Design and Implementation of Condition Monitoring System of Wind Turbine Gearbox
}

\author{
$\mathrm{Yu} \mathrm{Bu} u^{1, a}$ \\ ${ }^{1}$ School of Petroleum and Chemical Engineering, Dalian University of Technology, Dalian, 116000, \\ China \\ aemail
}

Keywords: Wind turbine gearbox, monitor system, condition monitoring

\begin{abstract}
The condition monitoring of wind power gearbox has important practical value for reducing maintenance cost and improving operation level. In this paper, a set of monitoring system for a wind turbine gearbox is designed. The condition monitoring system can monitor the vibration acceleration, speed and displacement of the wind turbine gearbox and generate monitoring reports. The experimental results show that the monitoring system can monitor the running state of the wind turbine gearbox in real time and has good reliability.
\end{abstract}

\section{Introduction}

The main function of the wind turbine gearbox is to transmit the power generated by the impeller under the influence of wind power to the generator and to obtain the corresponding speed. Gearbox is one of the most important parts of wind turbine. Its manufacturing technology is mature and has high reliability. The wind turbine condition monitoring technology adopts a variety of methods and means to monitor and analyze the important components of the unit on-line. We should find fault symptoms as early as possible to avoid and mitigate serious equipment damage. Although the gearbox fault rate is low, the maintenance process is complicated compared to the failure frequency and electric control system and the highest hydraulic system, especially for offshore wind turbines, ship and crane maintenance process requires special equipment and suitable weather. The gearbox downtime caused by all kinds of faults and maintenance costs are the highest. The gearbox of wind turbine has its own characteristics, that is, variable speed and variable load. With the change of wind speed, the load of gear box change at any time, which brings great challenge to the traditional condition monitoring method applied to the wind turbine gearbox. The cost performance of monitoring method should be considered when selecting the gear box condition monitoring method. Compared with the thermal power unit and the hydropower unit, the cost of the single typhoon machine is low, and the cost of the gearbox monitoring system must be taken into consideration. In order to ensure the safe and efficient operation of the wind turbine gearbox, it is necessary to strengthen the operation and maintenance of the equipment, make early diagnosis and prevention of the trouble, and eliminate the hidden trouble in time. The development health monitoring system of wind turbine gearbox can achieve the temperature, speed, vibration signal acquisition, processing and display, which has good application value.

\section{Hardware Design of Condition Monitoring System of Wind Turbine Gearbox}

In the monitoring area, the sensor collects temperature signals. At that time, the collected data is analog signal and will be converted into digital signal. The acquisition module can exist in either router node or terminal device node. The work of data processing module is to process the collected data through the processor and to operate the routing protocol, power management, task management and so on.

The host computer database server stores the data in real time and monitors the computer for real-time monitoring and data management. The fault characteristic value and the real-time monitoring result are stored in the server, which is convenient for monitoring and diagnosing the 
computer and calling the database history data. Server system mainly consists of background database, monitoring parameter acquisition, historical data query, client monitoring and response program. The client is mainly responsible for client request and response, historical data query, real-time display of vibration waveform and fault diagnosis, and other monitoring value display and alarm functions. The main task of wireless communication module is to exchange control messages and receive data between devices. The power management module provides electrical energy to the sensor nodes. In general, the coordinator is connected directly to the host without battery power.

The keyboard circuit is composed of direction key and command key. The direction key is completed by voltage sampling, and the key value is determined through different voltage values. This keyboard circuit is mainly realized under the program. The hardware circuit board can be achieved by different keys. In the download coordinator program, pressing the lower panel key of the attributes module can be defined as the network coordinator. Then, the system will start automatically to the network coordinator module. It will establish a network identity, display on the node to the coordinator and show it to the network. The data acquisition module has an analog multiplexer, which can support the most configurable input channels and realize multi-channel analog conversion. But it also has another input that can connect the data to the internal temperature sensor. The process does not require any intervention by the kernel. In this way, it is possible to ensure that a continuous sampling stream is captured.

\section{Software Design of Condition Monitoring System of Wind Turbine Gearbox}

Module of Data Acquisition. In the operation process, the surface of the rolling body is subjected to alternating load repeated action due to friction, lubrication, installation, maintenance and other aspects of the surface spalling. The bearing fracture is one of the common faults of gear box. The fundamental reason lies in the stress concentration factor was not eliminated in the manufacturing process of shaft under overload or alternating stress. The fatigue limit of the bearing force exceeds the material will lead to broken bearing. When the equipment fails, there will be certain characteristics, such as electrical signals, acceleration, speed, displacement, temperature, pressure, noise and so on. Through the analysis of these signals, the fault location and fault types of the equipment are determined. When the equipment is out of order, the vibration information can be reflected in the vibration signal. Therefore, through the vibration monitoring of the wind turbine generator equipment, we can observe the operation characteristics and parameters of the equipment failure. In addition, through the monitoring and collection of the temperature in the operation environment, the operation of the observation equipment can be assisted. In the operation of gear box transmission system, vibration is caused by the excitation of internal and external alternating loads. When the component fails, the mechanism changes, and the vibration of the structure will change accordingly. Vibration acceleration sensors are used to measure the vibration signals of each part of the gear box, and the fault characteristic information of the vibration signals is extracted. We use the DAQrmc driver to prepare acquisition program since the collected signal, error output and the output end are connected with the stop button Once the operation fails or the user presses the stop button, the program will immediately jump out of the while loop, stop data acquisition. The start of the channel takes precedence over the analog input channel.

Module of Condition Display. In this process, the first action is initialization of the coordinator hardware and protocol stack. If we can establish the network successfully, the new node is added. It is to distribute the address for the new node. We can realize the coordinator node and bound to win back the new node detection network. If it is successful, we begin to send and receive data through the serial port, then the coordinator will collect the information into the real-time display of the computer, and the data will be collected to store historical query. We should initialize the hardware and operating system and protocol for detection no matter whether there is a wireless network. Then, we should apply it to join the network. Once from other equipment to send data, we begin to receive data or forward. The overview monitoring refers to all measuring points on the selected machine, including the temperature measuring points, pressure measuring point and measuring point vibration 
measurements should be displayed in a table, the system responsible for the task of online dynamic display of real-time monitoring data, monitoring personnel convenient to grasp of global machine. Online monitoring is responsible for the historical trend display of monitoring data of each monitoring point. The user can make a preliminary judgment on the basic operation of the monitored machine or equipment according to the historical trend diagram, which includes the vibration trend analysis, temperature trend analysis, pressure trends and other functional modules. When the temperature value or the time frequency domain energy exceeds the set range, the corresponding indicator light will go on and continue to flicker. When the real time temperature value is greater than the temperature of the upper limit set value, the alarm indicator light flashes alternating red and green corresponding to the temperature; when a numeric temperature setting value is lower than the upper and higher than the lower limit, the normal temperature indicator light will light up continuously.

\section{Implementation of Condition Monitoring System of Wind Turbine Gearbox}

Data Acquisition. The condition monitoring system of wind turbine gearbox is designed to improve the ability of enterprise supervision and ensure the safe operation of the unit as the ultimate goal. Therefore, the system itself requires good reliability and stability. The developed state monitoring and fault diagnosis system is tested and verified on the gear box test bench of our laboratory. The layout of the test bench is shown in the diagram. The equipment specifications and parameter configuration of the test stand are shown in the table. The data acquisition module verifies that the vibration status of the gear box is collected and monitored during the test process. The vibration signals under no-load and exciting currents are collected and monitored respectively. To prevent external electric field or magnetic field interference, the tested signal lines are shielded. The main interface of the data acquisition module is shown in the Figure 1.

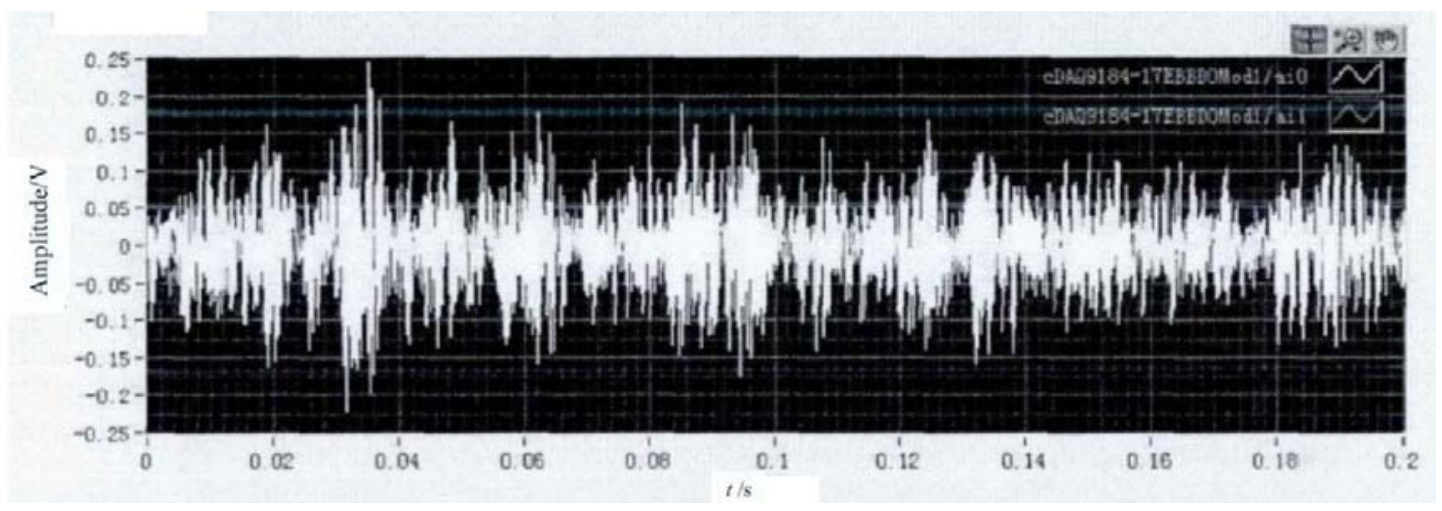

Figure 1. Main interface of the data acquisition module

Condition Display. The spectrum of the original vibration signal is analyzed, and the vibration spectrum of gear box is obtained when no-load and loaded. The maximum frequency of vibration occurs around, which coincides with the frequency of gear injection. The test bench is collected long time continuous vibration signal of the gear box, and the database is running well. The data acquisition module can accurately achieve continuous acquisition and monitoring function. Vibration feature extraction analysis based on singular value decomposition can verify that the feasibility of vibration signal feature extraction method is proposed. The method of gearbox vibration signal acquisition section is analyzed. The gear shaft speed is the sampling frequency and a section of data length is intercepted to analyze. In the case of lower order decomposition, singular value distribution curve of relative acts whistle. It is hard to choose the appropriate separation order with the increase of the order decomposition. The singular value distribution is gradually stepped, reflecting the gradually emerged useful signal singular value. At this point, it is easy to choose the proper order of separation for singular value decomposition. The main interface of the condition display module is shown in Figure 2. After testing and verification, the state monitoring system framework can meet the 
requirements of data acquisition and monitoring. The results show that condition display module has good reliability.

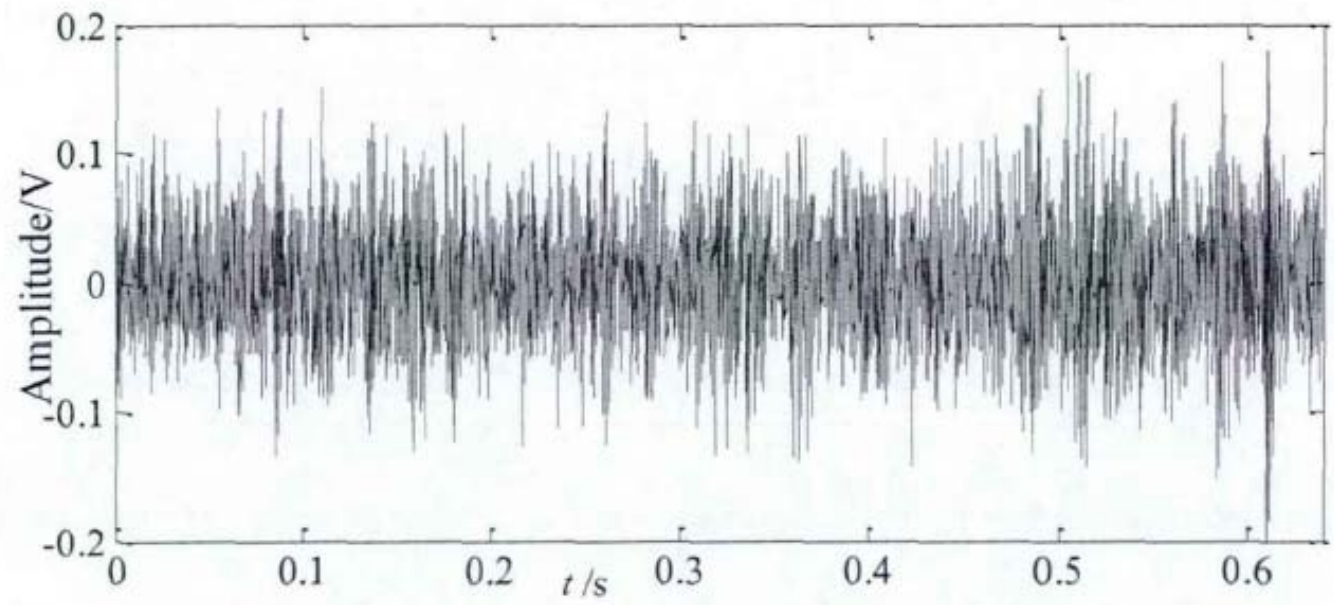

Figure 2. Main interface of the condition display module

\section{Conclusion}

The scheme of on-line monitoring system for wind turbine gearbox includes the data acquisition module and the condition display module. It can analyze the measured data in time and judge whether the equipment is faulty or not. The function of the on-line monitoring system of the wind turbine gear box is verified through the experiment. The results show that condition monitoring system of wind turbine gearbox achieves the desired function and has good stability.

\section{References}

[1] Wang Jianguo, Sun Xiaoming, Wang Shaofeng, Nan Kaigang, Health Condition Monitoring System Design for Wind Turbine Gear Box Based on LabVIEW [J]. Measurement \& Control Technology, 2017, 36(3): 119-122+131.

[2] Mi Lin, Feng Lengyu, Tan Wei, Project Design of On-Line Condition Monitoring System of Wind Turbine Gearbox [J]. Journal of Chongqing University of Technology (Natural Science), 2016, 30(2): 109-113.

[3] Sun Jianping, Zhu Wen, Zhai Yongjie, Ge Jianhong, Wind Turbine Gearbox Prognostic Simulation Research Based on MSET Method [J]. Journal of System Simulation, 2013, 25(12): 3009-3014.

[4] Guo Peng, David Infield, Yang Xiyun, Wind Turbine Gearbox Condition Monitoring Using Temperature Trend Analysis [J]. Proceedings of the CSEE, 2011, 31(15): 129-136. 